\title{
AXIOMATIC DIFFERENTIAL GEOMETRY I-1 - TOWARDS MODEL CATEGORIES OF DIFFERENTIAL GEOMETRY
}

\author{
HIROKAZU NISHIMURA
}

\begin{abstract}
In this paper we give an axiomatization of differential geometry comparable to model categories for homotopy theory. Weil functors play a predominant role.
\end{abstract}

\section{INTRODUCTION}

It is well known that the category of topological spaces and continuous mappings is by no means cartesian closed, which has harassed algebraic topologists. In 1967 Steenrod [21] popularized the idea of convenient category by announcing that the category of compactly generated spaces and continuous mappings renders a good setting for algebraic topology. The advertised category is cartesian closed, complete and cocomplete, and contains all CW complexes. In the same year, Quillen [15] finally succeeded in axiomatizing homotopy theory, which is now known as model categories.

Turning to differential geometry, more than a few geometers have tried to give a convenient category for differential geometry, e.g., [2], [3], [4], [5], [6], [7], [11], [14], [17], [18] and [19]. Some acute mathematicians compared these proposed convenient categories, e.g., [1] and [20]. Now what is completely missing is an axiomatization of differential geometry comparable to model categories for homotopy theory. We hastily home in on one so as to fill in the rift, at least as far as infinitesimal aspects of differential geometry are concerned.

Weil algebras were introduced by Weil himself [22]. They were intended for the algebraic realization of fabulous nilpotent infinitesimals. It is presumably synthetic differential geometers who have used Weil algebras systematically in differential geometry for the first time. They prefer to enjoy tangled relations among various Weil algebras. In particular, they have reached the crucial notion of microlinearity. To synthetic differential geometers, Weil functors are merely the exponentiation by infinitesimal objects corresponding to Weil algebras, while, to orthodox differential geometers, they are a natural generalization of the tangent bundle functor so that they can be defined without any reference to legendary infinitesimal objects. Roughly speaking, our axiomatization of differential geometry is a convenient category endowed with Weil functors. Generally speaking, any proposed convenient category is so broad as to contain spaces which are not necessarily amenable to

$M S C$ (2010): primary 53Z99,18F99.

Keywords: axiomatic differential geometry, Weil functor, model category, convenient category. 
the study by methods of differential geometry. From our standpoint, the notion of manifold is a flawed concept, or politely said, a transitory concept to be replaced by another more appropriate one, just as Riemann integrals were to be replaced by Lebesgue integrals. It is the notion of microlinearity that enables us to delineate the class of spaces adequate for the study of differential geometry. It gives us a great pleasure to see that the full subcategory of the convenient category consisting of all spaces susceptible of differential-geometric investigation is cartesian closed, whatever the convenient category may be. We will discuss our axiomatization in Section 3.

In orthodox differential geometry, just as smooth manifolds are spaces which are locally Euclidean (namely, locally diffeomorphic to some open subsets of $\mathbf{R}^{m}$ ), fibered manifolds are locally the canonical projections $\mathbf{R}^{m+n} \rightarrow \mathbf{R}^{m}$. As Mangiarotti and Modugno [13] have stressed, a large portion of differential geometry (at least up to connections and their related concepts) could be developed upon fibered manifolds. We should say that the orthodox notion of fibered manifold is slightly distorted, simply because the map is required to be a submersion so as to make every emerging entity amenable to the realm of manifolds. From our standpoint, the story goes as follows. Given a convenient category provided with Weil functors, its arrow category is also naturally endowed with derived Weil functors. Our notion of fiber bundle is simple enough. It is microlinear objects in the arrow category. This point will be discussed in detail in Section 4. In Section 5, we will discuss vertical Weil functors.

\section{Preliminaries}

\subsection{Category Theory}

There are many good textbooks on category theory. By way of example, [12] and [16] are recommendable classics. Therefore it would be absurd to try to explain category theory from scratch. However we must fix our own notation and terminology in this arena. A category $\mathcal{K}$ is called left exact if it has finite limits. A functor between left exact categories is called left exact if it preserves finite limits. A diagram in a category $\mathcal{K}$ is a functor $\mathcal{D}$ from a category $\Lambda$ to the category $\mathcal{K}$. Its limit in $\mathcal{K}$ is usually denoted by $\operatorname{Lim}_{\lambda \in \Lambda} \mathcal{D}_{\lambda}$. Given a natural transformation $\rho: \mathcal{J} \rightarrow \mathcal{K}$ between two functors $\mathcal{F}, \mathcal{G}: \mathcal{J} \rightarrow \mathcal{K}$ and an object $X$ in $\mathcal{J}$, the morphism $\mathcal{F}(X) \rightarrow \mathcal{G}(X)$ induced by the natural transformation $\rho$ is denoted by $\rho(X)$ or $\rho_{X}$. Given a category $\mathcal{K}$, its arrow category is usually denoted by $\overrightarrow{\mathcal{K}}$ in preference to $\mathcal{K} \rightarrow$.

\subsection{Weil Algebras}

Let $k$ be a commutative ring. The category of Weil algebras over $k$ (also called Weil $k$-algebras) is denoted by $\mathbf{W e i l}_{k}$. It is well known that the category $\mathbf{W e i l}_{k}$ is left exact. The terminal object in $\mathbf{W e i l}_{k}$ is $k$ itself, and, given an object $W$ in $\mathbf{W e i l}_{k}$, the unique morphism $W \rightarrow k$ in $\mathbf{W e i l}_{k}$ is denoted by $\underline{\tau}_{W}$. Since any object $W$ in Weil $_{k}$ is a $k$-algebra, there is a canonical morphism $k \rightarrow W$, which we denote by $\underline{\iota}_{W}$. Given two objects $W_{1}$ and $W_{2}$, we denote their tensor algebra by $W_{1} \otimes_{k} W_{2}$. For a good treatise on Weil algebras, the reader is referred to 
$\S 1.16$ of [9]. Given a left exact category $\mathcal{K}$ and a $k$-algebra object $\mathbb{R}$ in $\mathcal{K}$, there is a canonical functor $\mathbb{R} \otimes \cdot$ (denoted by $\mathbb{R} \otimes \cdot$ in [9]) from the category Weil . $_{k}$ to the category of $k$-algebra objects and their homomorphisms in $\mathcal{K}$.

\section{Axiomatics}

Definition 3.1. A DG-category (DG stands for Differential Geometry) is a quadruple $(\mathcal{K}, \mathbb{R}, \mathbf{T}, \alpha)$ where

(1) $\mathcal{K}$ is a category which is left exact and cartesian closed.

(2) $\mathbb{R}$ is a commutative $k$-algebra object in $\mathcal{K}$.

(3) Given a Weil $k$-algebra $W, \mathbf{T}^{W}: \mathcal{K} \rightarrow \mathcal{K}$ is a left exact functor for any Weil $k$-algebra $W$ subject to the condition that $\mathbf{T}^{k}: \mathcal{K} \rightarrow \mathcal{K}$ is the identity functor while we have

$$
\mathbf{T}^{W_{2}} \circ \mathbf{T}^{W_{1}}=\mathbf{T}^{W_{1} \otimes_{k} W_{2}}
$$

for any Weil $k$-algebras $W_{1}$ and $W_{2}$.

(4) Given a Weil $k$-algebra $W$, we have

$$
\mathbf{T}^{W} \mathbb{R}=\mathbb{R} \underline{\otimes} W .
$$

(5) $\alpha_{\varphi}: \mathbf{T}^{W_{1}} \rightarrow \mathbf{T}^{W_{2}}$ is a natural transformation for any morphism $\varphi: W_{1} \rightarrow$ $W_{2}$ in the category $\mathbf{W e i l}_{k}$ such that we have

$$
\alpha_{\psi} \cdot \alpha_{\varphi}=\alpha_{\psi \circ \varphi}
$$

for any morphisms $\varphi: W_{1} \rightarrow W_{2}$ and $\psi: W_{2} \rightarrow W_{3}$ in the category Weil ${ }_{k}$ while we have

$$
\alpha_{\mathrm{id}_{W}}=\mathrm{id}_{\mathbf{T}^{W}}
$$

for any identity morphism $\operatorname{id}_{W}: W \rightarrow W$ in the category $\mathbf{W e i l}_{k}$.

(6) Given a morphism $\varphi: W_{1} \rightarrow W_{2}$ in the category $\mathbf{W e i l}_{k}$, we have

$$
\alpha_{\varphi}(\mathbb{R})=\mathbb{R} \underline{\otimes} \varphi .
$$

Now some comments on the above definition are in order.

\section{Remark 3.2.}

(1) How far the category $\mathcal{K}$ should be exact is undoubtedly disputable. Every geometer with the seven cardinal virtues agrees that the category of smooth manifolds and smooth mappings is by no means exact enough. However the requirement that $\mathcal{K}$ should be a topos would presumably be demanding too much so long as $\mathcal{K}$ is expected to be naturally realizable in our real world. Synthetic differential geometers have constructed their well-adapted models, which are toposes, in their favotite imaginary world. Our requirement in this paper that $\mathcal{K}$ should be left exact and cartesian closed is barely minimal without doubt. This point will be discussed further in subsequent papers.

(2) The functors $\mathbf{T}^{W}$ 's stand for so-called Weil functors.

(3) The conditions 4 and 6 in the above definition correspond in a sense to (abstract) Taylor expansion theorem in calculus or to what is dubbed the (generalized) Kock-Lawvere axiom in synthetic differential geometry. 
(4) The formula (3.1) has been inspired by Proposition in 35.18 of [10].

(5) What is to be called the integration axiom should undoubtedly be considered. This point will be discussed in subsequent papers.

Notation 3.3. The natural transformation $\alpha_{\underline{\tau}_{W}}: \mathbf{T}^{W} \rightarrow \operatorname{id}_{\mathcal{K}}$ is denoted by $\tau_{W}$.

Notation 3.4. The natural transformation $\alpha_{\underline{\iota}_{W}}: \mathrm{id}_{\mathcal{K}} \rightarrow \mathbf{T}^{W}$ is denoted by $\iota_{W}$.

It is easy to see (cf. Chapter II, $\S 3$, Proposition 1 of [12]) that

Proposition 3.5. Given a DG-category $(\mathcal{K}, \mathbb{R}, \mathbf{T}, \alpha)$, the pair $(\mathbf{T}, \alpha)$ defines a bifunctor $\otimes_{\mathbf{T}, \alpha}: \mathcal{K} \times \mathbf{W e i l}_{k} \rightarrow \mathcal{K}$ in the sense that we have

$$
X \otimes_{\mathbf{T}, \alpha} W=\mathbf{T}^{W} X
$$

for any object $X$ in the category $\mathcal{K}$ and any Weil $k$-algebra $W$ while we have

$$
\begin{aligned}
& f \otimes_{\mathbf{T}, \alpha} \varphi \\
= & \alpha_{\varphi, Y} \circ \mathbf{T}^{W_{1}} f \\
= & \mathbf{T}^{W_{2}} f \circ \alpha_{\varphi, X}
\end{aligned}
$$

for any morphism $f: X \rightarrow Y$ in the category $\mathcal{K}$ and any morphism $\varphi: W_{1} \rightarrow W_{2}$ in the category $\mathbf{W e i l}_{k}$.

Notation 3.6. We will often write $X \otimes W$ in place of $X \otimes_{\mathbf{T}, \alpha} W$ unless any confusion may occur.

We shall fix a DG-category $(\mathcal{K}, \mathbb{R}, \mathbf{T}, \alpha)$ throughout the rest of the paper.

Definition 3.7. An object $X$ in the category $\mathcal{K}$ is called Weil exponentiable if

$$
\left(X \otimes\left(W_{1} \otimes_{k} W_{2}\right)\right)^{Y}=\left(X \otimes W_{1}\right)^{Y} \otimes W_{2}
$$

holds naturally for any object $Y$ in the category $\mathcal{K}$ and any Weil $k$-algebras $W_{1}$ and $W_{2}$.

Remark 3.8. If $Y=1$, then (3.2) degenerates into

$$
X \otimes\left(W_{1} \otimes_{k} W_{2}\right)=\left(X \otimes W_{1}\right) \otimes W_{2} .
$$

If $W_{1}=k$, then (3.2) degenerates into

$$
\left(X \otimes W_{2}\right)^{Y}=X^{Y} \otimes W_{2} .
$$

Proposition 3.9. If $X$ is a Weil exponentiable object in the category $\mathcal{K}$, then so is $X \otimes W$ for any Weil k-algebra $W$.

Proof. For any object $Y$ in the category $\mathcal{K}$ and any Weil $k$-algebras $W_{1}$ and $W_{2}$, we have

$$
\begin{aligned}
& \left((X \otimes W) \otimes\left(W_{1} \otimes_{k} W_{2}\right)\right)^{Y} \\
= & \left(X \otimes\left(\left(W \otimes_{k} W_{1}\right) \otimes_{k} W_{2}\right)\right)^{Y} \\
= & \left(X \otimes\left(W \otimes_{k} W_{1}\right)\right)^{Y} \otimes W_{2} \\
= & \left((X \otimes W) \otimes W_{1}\right)^{Y} \otimes W_{2}
\end{aligned}
$$

so that we have the desired result. 
Proposition 3.10. If $\mathcal{F}: \Lambda \rightarrow \mathcal{K}$ is a finite diagram in the category $\mathcal{K}$ such that $\mathcal{F}_{\lambda}$ is Weil exponentiable for any $\lambda \in \Lambda$, then $\operatorname{Lim}_{\lambda \in \Lambda} \mathcal{F}_{\lambda}$ is Weil exponentiable.

Proof. Since functors $\mathbf{T}^{W}: \mathcal{K} \rightarrow \mathcal{K}\left(\forall W \in \mathbf{W e i l}_{k}\right)$ and the exponentiation by $Y$ are left exact functors, we have

$$
\begin{aligned}
& \left(\left(\operatorname{Lim}_{\lambda \in \Lambda} \mathcal{F}_{\lambda}\right) \otimes\left(W_{1} \otimes_{k} W_{2}\right)\right)^{Y} \\
= & \left(\operatorname{Lim}_{\lambda \in \Lambda}\left(\mathcal{F}_{\lambda} \otimes\left(W_{1} \otimes_{k} W_{2}\right)\right)\right)^{Y} \\
= & \operatorname{Lim}_{\lambda \in \Lambda}\left(\mathcal{F}_{\lambda} \otimes\left(W_{1} \otimes_{k} W_{2}\right)\right)^{Y} \\
= & \operatorname{Lim}_{\lambda \in \Lambda}\left(\left(\mathcal{F}_{\lambda} \otimes W_{1}\right)^{Y} \otimes W_{2}\right) \\
= & \left(\operatorname{Lim}_{\lambda \in \Lambda}\left(\mathcal{F}_{\lambda} \otimes W_{1}\right)^{Y}\right) \otimes W_{2} \\
= & \left(\operatorname{Lim}_{\lambda \in \Lambda}\left(\mathcal{F}_{\lambda} \otimes W_{1}\right)\right)^{Y} \otimes W_{2} \\
= & \left(\left(\operatorname{Lim}_{\lambda \in \Lambda} \mathcal{F}_{\lambda}\right) \otimes W_{1}\right)^{Y} \otimes W_{2}
\end{aligned}
$$

so that we have the desired result.

Proposition 3.11. If $X$ is a Weil exponentiable object in the category $\mathcal{K}$, then so is $X^{Y}$ for any object $Y$ in the category $\mathcal{K}$.

Proof. For any object $Z$ in category $\mathcal{K}$ and any Weil $k$-algebras $W_{1}$ and $W_{2}$, we have

$$
\begin{aligned}
& \left(X^{Y} \otimes\left(W_{1} \otimes_{k} W_{2}\right)\right)^{Z} \\
= & \left(X \otimes\left(W_{1} \otimes_{k} W_{2}\right)\right)^{Y \times Z} \\
= & \left(X \otimes W_{1}\right)^{Y \times Z} \otimes W_{2} \\
= & \left(\left(X \otimes W_{1}\right)^{Y}\right)^{Z} \otimes W_{2} \\
= & \left(X^{Y} \otimes W_{1}\right)^{Z} \otimes W_{2}
\end{aligned}
$$

so that we have the desired result.

Theorem 3.12. The full subcategory $\mathcal{K}_{\mathbf{W E}}$ of all Weil exponentiable objects in the category $\mathcal{K}$ is a left exact and cartesian closed category.

Proof. This follows simply from Propositions 3.10 and 3.11 .

Definition 3.13. An object $X$ in the category $\mathcal{K}$ is called microlinear providing that any finite limit diagram $\mathcal{D}$ in the category Weil $_{k}$ yields a limit diagram $X \otimes \mathcal{D}$ in $\mathcal{K}$ where $X \otimes \mathcal{D}$ is obtained from $\mathcal{D}$ by putting $X \otimes$ to the left of every object and every morphism in $\mathcal{D}$.

Proposition 3.14. If an object $X$ in the category $\mathcal{K}$ is Weil exponentiable and microlinear, then so is $X \otimes W$ for any Weil k-algebra $W$.

Proof. Given a finite limit diagram $\mathcal{D}$ in the category $\mathbf{W e i l}_{k}$, we have

$$
(X \otimes W) \otimes \mathcal{D}=X \otimes\left(W \otimes_{k} \mathcal{D}\right)
$$

by (3.3). Since the functor $W \otimes_{k} \cdot: \mathbf{W e i l}_{k} \rightarrow \mathbf{W e i l}_{k}$ preserves finite limits, we have the desired result. 
Proposition 3.15. If $\mathcal{F}: \Lambda \rightarrow \mathcal{K}$ is a finite diagram in the category $\mathcal{K}$ such that $\mathcal{F}_{\lambda}$ is a microlinear object in $\mathcal{K}$ for any $\lambda \in \Lambda$, then its limit $\operatorname{Lim}_{\lambda \in \Lambda} \mathcal{F}_{\lambda}$ is also a microlinear object in $\mathcal{K}$.

Proof. Given a finite diagram $\mathcal{D}: \Gamma \rightarrow \mathbf{W e i l}_{k}$ in the category $\mathbf{W e i l}_{k}$, we have

$$
\begin{aligned}
& \operatorname{Lim}_{\gamma \in \Gamma} \quad\left(\left(\operatorname{Lim}_{\lambda \in \Lambda} \mathcal{F}_{\lambda}\right) \otimes \mathcal{D}_{\gamma}\right) \\
= & \operatorname{Lim}_{\gamma \in \Gamma} \quad\left(\operatorname{Lim}_{\lambda \in \Lambda} \quad\left(\mathcal{F}_{\lambda} \otimes \mathcal{D}_{\gamma}\right)\right) \\
= & \operatorname{Lim}_{\lambda \in \Lambda} \quad\left(\operatorname{Lim}_{\gamma \in \Gamma} \quad\left(\mathcal{F}_{\lambda} \otimes \mathcal{D}_{\gamma}\right)\right) \\
& {[\text { since double limits commute }] } \\
= & \operatorname{Lim}_{\lambda \in \Lambda} \quad\left(\mathcal{F}_{\lambda} \otimes\left(\operatorname{Lim}_{\gamma \in \Gamma} \mathcal{D}_{\gamma}\right)\right) \\
& {\left[\operatorname{since}_{\mathcal{F}_{\lambda}} \text { is microlinear }\right] } \\
= & \left(\operatorname{Lim}_{\lambda \in \Lambda} \mathcal{F}_{\lambda}\right) \otimes\left(\operatorname{Lim}_{\gamma \in \Gamma} \mathcal{D}_{\gamma}\right)
\end{aligned}
$$

so that we have the desired result.

Proposition 3.16. If $X$ is a Weil exponentiable and microlinear object in $\mathcal{K}$, then so is $X^{Y}$ for any object $Y$ in $\mathcal{K}$.

Proof. $X^{Y}$ is Weil exponentiable by Proposition 3.11. Given a finite diagram $\mathcal{D}: \Gamma \rightarrow$ Weil $_{k}$, we have

$$
\begin{aligned}
& \operatorname{Lim}_{\gamma \in \Gamma}\left(X^{Y} \otimes \mathcal{D}_{\gamma}\right) \\
= & \operatorname{Lim}_{\gamma \in \Gamma}\left(X \otimes \mathcal{D}_{\gamma}\right)^{Y} \\
& {[\text { by }(3.4)] } \\
= & \left(\operatorname{Lim}_{\gamma \in \Gamma}\left(X \otimes \mathcal{D}_{\gamma}\right)\right)^{Y} \\
& {[\text { since the exponentiation by } Y \text { is a left exact functor }] } \\
= & \left(X \otimes\left(\operatorname{Lim}_{\gamma \in \Gamma} \mathcal{D}_{\gamma}\right)\right)^{Y} \\
& {[\text { since } X \text { is microlinear }] } \\
= & X^{Y} \otimes\left(\operatorname{Lim}_{\gamma \in \Gamma} \mathcal{D}_{\gamma}\right) \\
& {[\text { by }(3.4)] }
\end{aligned}
$$

so that $X^{Y}$ is microlinear.

Now we recapitulate as follows.

Theorem 3.17. The full subcategory $\mathcal{K}_{\mathbf{W E}, \mathbf{M L}}$ of all Weil exponentiable and microlinear objects in the category $\mathcal{K}$ is a left exact and cartesian closed category.

\section{Fibered Microlinear Objects}

Now we are going to talk about fibered manifolds in our context. First of all, we note that

Proposition 4.1. The quadruple $(\overrightarrow{\mathcal{K}}, \overrightarrow{\mathbb{R}}, \overrightarrow{\mathbf{T}}, \vec{\alpha})$ is a DG-category where

(1) $\overrightarrow{\mathcal{K}}$ is the arrow category of $\mathcal{K}$. 
(2) $\overrightarrow{\mathbb{R}}$ stands for

$$
\begin{aligned}
& \mathbb{R} \\
& \downarrow \\
& 1
\end{aligned}
$$

(3) Given a Weil k-algebra $W, \overrightarrow{\mathbf{T}}^{W}\left(\begin{array}{c}E \\ \pi \\ M\end{array}\right)$ is

$$
\begin{gathered}
\mathbf{T}^{W}(E) \\
\mathbf{T}^{W}(\pi) \downarrow \\
\mathbf{T}^{W}(M)
\end{gathered}
$$

$$
\begin{aligned}
& \text { while } \overrightarrow{\mathbf{T}} W\left(\begin{array}{ccc}
E_{1} & f & E_{2} \\
\pi_{1} \downarrow & & \downarrow \pi_{2} \\
M_{1} & \bar{f} & M_{2}
\end{array}\right) \text { is }
\end{aligned}
$$



(4) Given a morphism $\varphi: W_{1} \rightarrow W_{2}$ in the category $\mathbf{W e i l}_{k}, \vec{\alpha}_{\varphi}\left(\begin{array}{c}E \\ \pi \\ M\end{array}\right)$ is

$$
\begin{array}{cccc}
\mathbf{T}^{W_{1}}(E) & & \alpha_{\varphi}(E) & \mathbf{T}^{W_{2}}(E) \\
\mathbf{T}^{W_{1}}(\pi) \downarrow & & \downarrow & \mathbf{T}^{W_{2}}(\pi) \\
\mathbf{T}^{W_{1}}(M) & \overrightarrow{\alpha_{\varphi}(M)} & \mathbf{T}^{W_{2}}(M)
\end{array} .
$$

Proof. That the category $\overrightarrow{\mathcal{K}}$ is left exact and that the functor $\overrightarrow{\mathbf{T}}^{W}: \overrightarrow{\mathcal{K}} \rightarrow \overrightarrow{\mathcal{K}}$ is left exact follow at the same time from Theorem 7.5.2 in [16]. That the category $\overrightarrow{\mathcal{K}}$ is cartesian closed follows from Exercise 1.3.7 in [8]. The other conditions for $(\overrightarrow{\mathcal{K}}, \overrightarrow{\mathbb{R}}, \overrightarrow{\mathbf{T}}, \vec{\alpha})$ to be a DG-category are easy to verify.

Corollary 4.2. ${ }_{M}^{E}{ }_{M}^{\downarrow} \in \overrightarrow{\mathcal{K}}$ is microlinear with respect to the DG-category $(\overrightarrow{\mathcal{K}}, \overrightarrow{\mathbb{R}}, \overrightarrow{\mathbf{T}}, \vec{\alpha})$ iff both $E$ and $M$ are microlinear with respect to the DG-category $(\mathcal{K}, \mathbb{R}, \mathbf{T}, \alpha)$.

Remark 4.3. Given two objects $\pi: E \rightarrow M$ and $\theta: F \rightarrow N$ in the category $\overrightarrow{\mathcal{K}}$, their exponential $\pi^{\theta}:\left(E^{F}\right)_{\mathbf{P}} \rightarrow M^{N}$ is determined by the pullback diagram

$$
\begin{array}{ccc}
\left(E^{F}\right)_{\mathbf{P}} & \rightarrow & E^{F} \\
\downarrow & & \downarrow \\
M^{N} & \rightarrow & M^{F}
\end{array}
$$

where $\mathbf{P}$ stands for "Projectable (into $M^{N}$ )". 
Proposition 4.4. Given a morphism $\pi: E \rightarrow M$ in the category $\mathcal{K}$, if both $E$ and $M$ are Weil exponentiable as objects in the category $\mathcal{K}$, then $\pi: E \rightarrow M$ is Weil exponentiable as an object in the category $\overrightarrow{\mathcal{K}}$.

Proof. As we have noted, $\left(\left(E \otimes W_{1}\right)^{F}\right)_{\mathbf{P}}$ is obtained as the pullback of the diagram

$$
\begin{array}{ccc}
\left(\left(E \otimes W_{1}\right)^{F}\right)_{\mathbf{P}} & \rightarrow & \left(E \otimes W_{1}\right)^{F} \\
\downarrow & & \downarrow \\
\left(M \otimes W_{1}\right)^{N} & \rightarrow & \left(M \otimes W_{1}\right)^{F}
\end{array}
$$

Since the functor $\otimes W_{2}: \mathcal{K} \rightarrow \mathcal{K}$ is left exact, the diagram obtained from (4.1) by the application of the functor

$$
\begin{array}{cccc}
\left(\left(E \otimes W_{1}\right)^{F}\right)_{\mathbf{P}} \otimes W_{2} \rightarrow \rightarrow & \left(E \otimes W_{1}\right)^{F} \otimes W_{2} \\
\downarrow & & \left(E \otimes\left(W_{1} \otimes_{k} W_{2}\right)\right)^{F} \\
\downarrow & \\
\downarrow & & \left(M \otimes W_{1}\right)^{F} \otimes W_{2} \\
\left(M \otimes W_{1}\right)^{N} \otimes W_{2} & & \rightarrow & \left(M \otimes\left(W_{1} \otimes_{k} W_{2}\right)\right)^{F}
\end{array}
$$

is a pullback diagram. However, the diagram

$$
\begin{array}{ccc}
\left(\left(E \otimes\left(W_{1} \otimes_{k} W_{2}\right)\right)^{F}\right)_{\mathbf{P}} & \rightarrow & \left(E \otimes\left(W_{1} \otimes_{k} W_{2}\right)\right)^{F} \\
\downarrow & \downarrow \\
\left(M \otimes\left(W_{1} \otimes_{k} W_{2}\right)\right)^{N} & \rightarrow & \left(M \otimes\left(W_{1} \otimes_{k} W_{2}\right)\right)^{F}
\end{array}
$$

is a pullback diagram. Therefore we have

$$
\left(\left(E \otimes\left(W_{1} \otimes_{k} W_{2}\right)\right)^{F}\right)_{\mathbf{P}}=\left(\left(E \otimes W_{1}\right)^{F}\right)_{\mathbf{P}} \otimes W_{2},
$$

which is the desired result.

Definition 4.5. By a fibered microlinear object in $\mathcal{K}$ we mean simply an object $\pi: E \rightarrow M$ in the category $\overrightarrow{\mathcal{K}}$ which is Weil exponentiable and microlinear with respet to the DG-structure $(\overrightarrow{\mathcal{K}}, \overrightarrow{\mathbb{R}}, \overrightarrow{\mathbf{T}}, \vec{\alpha})$.

Notation 4.6. The full subcategory of $\overrightarrow{\mathcal{K}}$ consisting of all fiber bundles in $\mathcal{K}$ is denoted by $\mathcal{K}_{\text {Fib }}$.

Theorem 4.7. The category $\mathcal{K}_{\mathbf{F i b}}$ is left exact and cartesian closed.

Proof. This follows directly from Theorem 3.17.

\section{Vertical Constructions}

Now we are going to discuss vertical bundles in our context.

Definition 5.1. Given a morphism $\pi: E \rightarrow M$ in the category $\mathcal{K}$ and a Weil $k$-algebra $W$, the vertical bundle $\tau_{W}^{\mathbf{V}}(\pi): \mathbf{V}^{W}(\pi) \rightarrow E$ of $\pi$ with respect to $W$ is defined to be

$$
\tau_{W}^{\mathbf{V}}(\pi)=\tau_{W}(E) \circ \widetilde{\tau}_{W}^{\mathbf{V}}(\pi)
$$


where $\widetilde{\tau}_{W}^{\mathbf{V}}(\pi): \mathbf{V}^{W}(\pi) \rightarrow E \otimes W$ is obtained as the equalizer of

$$
E \otimes W \underset{\pi \otimes \operatorname{id}_{W}}{\longrightarrow} M \otimes W
$$

and

$$
E \otimes W \stackrel{\tau_{W, E}}{\longrightarrow} \stackrel{\pi}{\longrightarrow} M \stackrel{\iota_{W, M}}{\longrightarrow} M \otimes W .
$$

Notation 5.2. We will often write $E \otimes^{\perp} W$ for $\mathbf{V}^{W}(\pi)$.

Lemma 5.3. Given a diagram in a left exact category $\mathcal{J}$

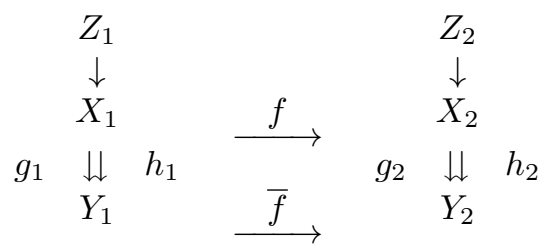

if both of the two diagrams
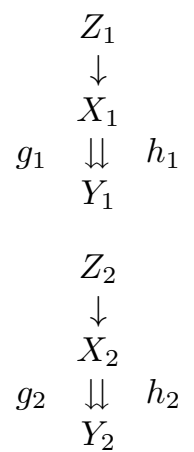

are equalizers, and if both of the diagrams

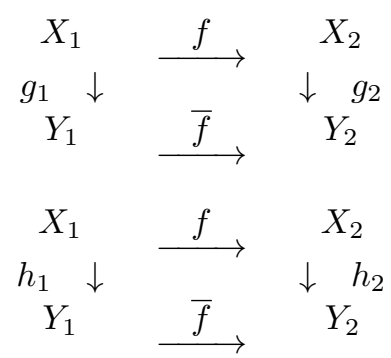

commute, then there is a unique morphism $Z_{1} \rightarrow Z_{2}$ making the diagram



commutative.

Proof. By the familiar token of what is dubbed arrow chasing. 
Corollary 5.4. Given a Weil k-algebra $W$, our previous mapping $\mathbf{V}^{W}$ assigning the object $\mathbf{V}^{W}(\pi)$ in the category $\mathcal{K}$ to each object

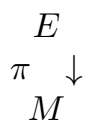

in the category $\overrightarrow{\mathcal{K}}$ can naturally be extended to a functor $\mathbf{V}^{W}: \overrightarrow{\mathcal{K}} \rightarrow \mathcal{K}$ in the sense that the diagram

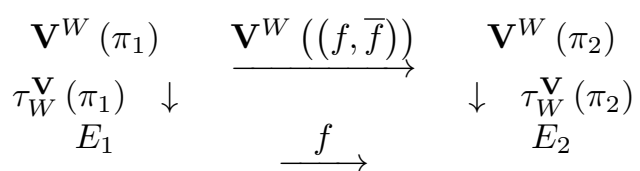

commutes for any morphism

in the category $\overrightarrow{\mathcal{K}}$.

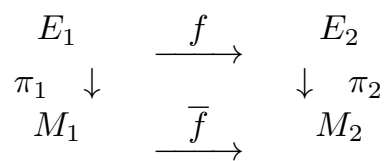

Proof. It suffices to note that

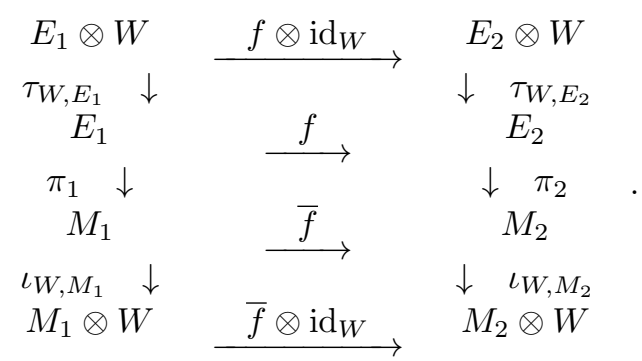

In particular, the outer rectangle is commutative, so that the desired result follows directly from the lemma.

Lemma 5.5. Given three finite diagrams $\mathcal{F}, \mathcal{G}, \mathcal{H}: \Lambda \rightarrow \mathcal{J}$ in a left exact category $\mathcal{J}$ with the same underlying category $\Lambda$, two natural transformations $\mu, \nu$ : $\mathcal{G} \rightarrow \mathcal{H}$ and a natural transformation $\theta: \mathcal{F} \rightarrow \mathcal{G}$, if the diagrams $\mathcal{G}$ and $\mathcal{H}$ are limit diagrams, and if the diagram

$$
\begin{array}{ccc} 
& \mathcal{F}_{\lambda} & \\
\theta_{\lambda} & \downarrow \\
& \mathcal{G}_{\lambda} & \\
\mu_{\lambda} \underset{\downarrow}{ } \stackrel{\downarrow}{\downarrow} \nu_{\lambda} \\
& \mathcal{H}_{\lambda} &
\end{array}
$$

is an equalizer for each $\lambda \in \Lambda$, then the diagram $\mathcal{F}$ is a limit diagram.

Proof. By the familiar token of what is called arrow chasing.

Corollary 5.6. Given a Weil k-algebra $W$, the functor $\mathbf{V}^{W}: \overrightarrow{\mathcal{K}} \rightarrow \mathcal{K}$ is left exact. 
Proof. Given a finite limit diagram in the category $\overrightarrow{\mathcal{K}}$, which decomposes into two limit diagrams $\mathcal{G}^{\prime}, \mathcal{H}^{\prime}: \Lambda \rightarrow \mathcal{K}$ in the category $\mathcal{K}$ and a natural transformation $\mu^{\prime}: \mathcal{G}^{\prime} \rightarrow \mathcal{H}^{\prime}$, we have two limit diagrams

$$
\begin{aligned}
\mathcal{G} & =\mathcal{G}^{\prime} \otimes W \\
\mathcal{H} & =\mathcal{H}^{\prime} \otimes W
\end{aligned}
$$

and two natural transformations

$$
\begin{aligned}
& \mu=\mu^{\prime} \otimes W: \mathcal{G} \rightarrow \mathcal{H} \\
& \nu: \mathcal{G} \overrightarrow{\tau_{W}} \mathcal{G}^{\prime} \overrightarrow{\mu^{\prime}} \mathcal{H}^{\prime} \dot{\vec{\iota}} \mathcal{H} .
\end{aligned}
$$

Therefore the desired result follows from the lemma.

Corollary 5.7 (The Vertical Microlinearity Theorem). Let $\pi: E \rightarrow M$ be a morphism in the category $\mathcal{K}$ with $E$ and $M$ being microlinear. If $\mathcal{D}$ is a finite limit diagram in the category $\mathbf{W e i l}_{k}$, then $E \otimes^{\perp} \mathcal{D}$ is a limit diagram in the category $\mathcal{K}$.

Proof. It suffices to note that both $E \otimes \mathcal{D}$ standing for $\mathcal{G}$ in the above lemma and $M \otimes \mathcal{D}$ standing for $\mathcal{H}$ in the above lemma are limit diagrams because $E$ and $M$ are assumed to be microlinear. Then the desired result follows directly from the lemma.

\section{REFERENCES}

[1] J. C. Baez and A. E. Hoffnung, Convenient categories of smooth spaces, Trans. Amer. Math. Soc. 363 (2011), 5789-5825.

[2] K. T. Chen, Iterated integrals of differential forms and loop space homology, Ann. of Math. 97 (1973), 249-268.

[3] K. T. Chen, Iterated integrals, fundamental groups and covering spaces, Trans. Amer. Math. Soc. 206 (1975), 83-98.

[4] K. T. Chen, Iterated path integrals, Bull. Amer. Math. Soc. 83 (1977), 831-879.

[5] A. Frölicher, Smooth structures, Lecture Notes in Mathematics, 962 (1982), 69-81, SpringerVerlag, Berlin and Heidelberg.

[6] A. Frölicher, Cartesian closed categories and analysis of smooth maps, Lecture Notes in Mathematics, 1174 (1986), 43-51, Springer-Verlag, Berlin and Heidelberg.

[7] A. Frölicher and A. Kriegl, Linear Spaces and Differentiation Theory, John Wiley and Sons, Chichester, 1988.

[8] B. Jacobs, Categorical Logic and Type Theory, Elsevier, Amsterdam, 1999.

[9] A. Kock, Synthetic Differential Geometry, 2nd ed., London Mathematical Society Lecture Note Series 333, Cambridge University Press, Cambridge, 2006.

[10] I. Kolář, P. W. Michor and J. Slovák, Natural Operations in Differential Geometry, SpringerVerlag, Berlin and Heidelberg, 1993.

[11] A. Kriegl and P. W. Michor, The Convenient Setting of Global Analysis, American Mathematical Society, Rhode Island, 1997.

[12] S. MacLane, Categories for the Working Mathematician, Springer, New York, 1971.

[13] L. Mangiarotti and M. Modugno, Connections and differential calculus on fibered manifolds, unpublished draft, 1989.

[14] M.A. Mostow, The differentiable structures of Milnor classifying spaces, simplicial complexes, and geometric realizations, J. Differential Geom. 14 (1979), 255-293.

[15] D. G. Quillen, Homotopical Algebra, Lecture Notes in Math. 43, Springer, Berlin, 1967.

[16] H. Schubert, Categories, Springer, Berlin Heidelberg, 1972.

[17] R. Sikorski, Differential modules, Colloq. Math. 24 (1971), 45-79. 
[18] J. W. Smith, The de Rham theorem for general spaces, Tôhoku Math. J. 18 (1966), 115-137.

[19] J. M. Souriau, Groupes differentiels, Lecture Notes in Math. 836, 91-128, Springer, Berlin Heidelberg, 1980.

[20] A. Stacey, Comparative smootheology, Theory and Applications of Categories 25 (2011), $64-117$.

[21] N. E. Steenrod, A convenient category of topological spaces, Michigan Math. J. 14 (1967), 133-152.

[22] A. Weil, Théorie des points proches sur les variétés différentiables, Colloques Internationaux du Centre National de la Reserche Scientifique, Strassbourg, 111-117, 1953.

Hirokazu Nishimura, Institute of Mathematics, University of Tsukuba, Tsukuba, Ibaraki, 305-8571, Japan

e-mail: logic@math.tsukuba.ac.jp 